\title{
PASADO Y PRESENTE DE LA ORIENTACIÓN EN VENEZUELA. INFLUENCIA ESPAÑOLA
}

\author{
PAST AND PRESENT OF EDUCATIONAL GUIDANCE IN VENEZUELA. \\ SPANISH INFLUENCE
}

\author{
Julia Boronat Mundina* y Denyzluz Molina Contreras** \\ U.N.E.D.* y U.N.E.LL.E.Z.**
}

\begin{abstract}
RESUMEN
Este artículo contempla el origen, desarrollo y situación actual de la orientación en un país latinoamericano: Venezuela. Cabe destacar la profunda influencia española en dos momentos muy significativos: El primero, contextualizado en la tercera década del siglo XX, cuando prestigiosos profesores españoles dejaron sentir su influencia en el campo educativo, incidiendo en la necesidad de contemplar la orientación profesional en este ámbito; el segundo, más reciente, en los años noventa, cuando el Ministerio de Educación de Venezuela toma como referente la Reforma educativa (LOGSE, 1990), ocupando la orientación escolar una presencia real y progresiva en su normativa, aplicada en los tres niveles curriculares, la formación de valores y los temas transversales.
\end{abstract}

Palabras clave: orientación educativa, sistema educativo venezolano, orientación profesional.

\section{ABSTRACT}

This paper deals with the origin, development, and present situation of guidance in a Latin-American country: Venezuela. It is possible to point out the deep Spanish influence in two moments of significant changes. One during the second decade of the $\mathrm{XX}^{\text {th }}$ century, when prestigious Spanish professors showed their influence in the educational field, by incorporating vocational guidance. The other one, more recent, during the nineties, when the Venezuelan educational system takes our reform (LOGSE, 1990) as reference, having school guidance a real and progressive

* Catedrática de Escuela Universitaria. Tiene diversas publicaciones enfocadas fundamentalmente hacia estos ámbitos: orientación escolar y profesional, educación infantil, docencia y orientación en la universidad, temáticas en torno a las cuales pivota su docencia e investigación. Profesora tutora del Centro Asociado de la UNED de Palencia.

** Profesora de la Facultad de Educación y coordinadora del proceso de evaluación de la UNELLEZ. Docencia e investigación en Orientación. Participa como especialista en el dirección y asesoramiento de diversos Proyectos Pedagógicos de la Escuela Básica. Cuenta con diversas publicaciones sobre la orientación personal-social y comunitaria. 
presence in the three levels of the Venezuelan curriculum, in the values education and in the transversal subjects.

Keywords: school guidance, Venezuelan educational system, tutorial system, vocational guidance.

\section{Introducción}

Para ubicar el tema de la orientación en el contexto venezolano, nos proponemos analizar dos factores que influyeron decisivamente en su génesis. Por una parte, la profunda influencia española y europea; y, por otra, los cambios derivados del paso de una sociedad precapitalista a una sociedad industrial, vinculada a la explotación y comercialización del petróleo.

Asimismo, examinamos de forma más detallada el devenir histórico de la orientación, impulsada desde los años sesenta por la incorporación de un modelo educativo liberal, que propició reformas notables en el sistema educativo venezolano, consolidado en 1974 con la denominada "Revolución Educativa", movimiento que enfatizó en la democratización de la enseñanza y en la modernización del proceso educativo.

Por último, nos detenemos en el marco normativo vigente de la orientación venezolana, fiel reflejo de la legislación educativa española; ambas inciden en el establecimiento de vínculos entre educación y orientación, y en la necesidad de incorporar la orientación en los centros educativos, haciéndola extensiva a todos los alumnos y agentes educativos. También reconocen la necesidad de contemplar la actividad orientadora paralelamente al desarrollo del currículo en los diferentes niveles educativos. Pero en el momento actual, en España se constata la perdida de protagonismo de la orientación en aspectos fundamentales y reconocidos anteriormente (Anteproyecto de Ley Orgánica de Educación; 2005), laguna que esperamos que sea subsanada, devolviéndole el lugar que le corresponde.

\section{Génesis y devenir histórico de la orientación venezolana}

El inicio de la orientación en Latinoamérica puede situarse en la década de los años treinta del siglo pasado. En sus primeros momentos, la orientación se aplicó al campo educativo de forma aislada y discontinua, al carecer de una infraestructura básica y de personal especializado para acometer este tipo de intervención. Los incipientes servicios, creados por iniciativa de algunos psicólogos, psiquiatras y médicos europeos, llegados de diversos países, reflejan una clara influencia europea, poniendo el acento en las tareas de selección y orientación profesional, con marcado enfoque psicométrico.

En la década de los cuarenta, la educación venezolana recibió una notoria influencia de profesionales exiliados de la Guerra Civil Española y de la Segunda Guerra Mundial, luctuosos sucesos que originaron que un número considerable de emigrantes fueran acogidos en los países latinoamericanos. Entre ellos, destacan el doctor José Ortega Durán, quien fundó en 1945 el Servicio de Psicotecnia en el Liceo Andrés Bello de Caracas; el profesor Miguel Antonio Aguirre, que creó el Laboratorio de Psicotecnia en la Escuela Normal; Francisco del Olmo, graduado en Bruselas como orientador; el profesor Vicente Costanzo, 
graduado en Pedagogía y Filosofía en la Universidad de Roma y el profesor Escalona, quien había cursado la especialidad junto al Dr. Mira y López, médico y director del Instituto de Orientación y Selección Profesional de Barcelona. Este último fijó definitivamente su residencia en Brasil, al igual que Joaquín Xirau se afincó en México, y Francisco del Olmo en Venezuela. Todos ellos influyeron decisivamente en la génesis de la orientación en los países latinoamericanos (Benavent, 1996).

Pero la implantación de un régimen militar en Venezuela, como también acaeció en España, ahogó las iniciativas innovadoras, sumiendo a la educación en un progresivo deterioro, que afectó también a la práctica de la orientación en el sistema educativo venezolano.

En 1958, como consecuencia del derrocamiento de la dictadura y el retorno del régimen democrático, se produce la movilización masiva de los campesinos venezolanos a las ciudades, debido al creciente desarrollo industrial y urbano. A raíz de la expansión de la industria petrolera, este país inicia el paso de la Venezuela "rural" a la "petrolera", fenómeno que trajo consigo la emigración de muchos trabajadores del campo hacia las ciudades, donde encontraban empleo más fácilmente. El alto grado de movilización de la población rural hacia las zonas industrializadas dio lugar a problemas de desarraigo, proliferación de enfermedades sexuales, y agudizó las carencias afectivas, sociales y culturales de las clases sociales menos favorecidas.

Este fenómeno coincide con la llegada al poder de Rómulo Betancourt en 1958, primer presidente electo democráticamente, periodo en que la Escuela Liberal alcanzó un significativo desarrollo. Los fundamentos sociales y la concepción ideológica de esta corriente está relacionada con los grupos politizados de las clases medias, quienes asumieron el papel de verdaderos agentes de cambio en esta época. De hecho, la burguesía industrial y los nuevos sectores urbanos en ascenso se opusieron a los proyectos aristocráticos de los terratenientes, reivindicando en el campo educativo una escuela obligatoria y laica.

En opinión de Calonge (1988:14), los cambios socioeconómicos operados en aquella época desembocaron en una concepción de la educación de carácter democrático, extensible a todos los sujetos, generando una amplia polémica con la iglesia, ante la educación selectiva que ésta promovía. Se vio la necesidad de incorporar una orientación enfocada hacia su dimensión vocacional y personal, tratando de abordar las situaciones conflictivas del contexto escolar.

El fortalecimiento de la clase media y, por ende, la incorporación del modelo educativo liberal, propició reformas notables en el sistema educativo. En efecto, se inicia un proceso de diversificación de la enseñanza, reflejo, a su vez, de la diversidad ocupacional y de la especialización en el trabajo. En esta situación, se constata la necesidad de la orientación, como un elemento que vincule las necesidades individuales de los educandos y las demandas globales de la sociedad.

Asimismo, el fenómeno de la emigración aceleró el crecimiento de la población y trajo consigo la creación de nuevos centros escolares, el ingreso de un mayor número de alumnos, la dotación de plazas docentes y la consiguiente proliferación de instituciones de formación del profesorado. Sin embargo, la creciente masificación de las aulas y la carencia de servicios de atención integral al alumno, derivaron en una serie de problemas como: elevados índices de repetición, deserción, bajo rendimiento y deterioro académico, entre otros, lacras que aún perviven en la Venezuela del siglo XXI. 
En los años sesenta, para atender las necesidades de la población escolarizada, el Ministerio de Educación promovió la creación de la División de Orientación del Ministerio de Educación (DOME) y, paralelamente, inició la formación de orientadores. De hecho, en 1962 se produjo la reorganización de los Centros, Institutos y Servicios de Psicopedagogía en las grandes ciudades, como Maracaibo, Barquisimeto y Caracas. En esta ciudad se fundó el Departamento de Orientación y Bienestar Estudiantil, dependiente del Instituto Experimental de Formación Docente. Como Servicio específico de Orientación, se creó el Instituto Pedagógico Experimental de Barquisimeto, presidido por estos principios:

- Los Servicios de orientación deben ofrecerse a todos los alumnos.

- La orientación atiende todos los aspectos de la personalidad del sujeto.

- La orientación se concibe como un proceso integrado en la enseñanza.

Este enfoque, ciertamente novedoso en su época, trataba de ofrecer asesoría y ayuda al alumno en todos sus dimensiones, superando la concepción remedial-asistencial de la orientación, como la únicamente válida. Sin embargo, a pesar de los esfuerzos realizados, esta propuesta tuvo un carácter eminentemente teórico, pues aunque estaba contemplada en diversas normativas, no pudo aplicarse en el contexto escolar, debido a la carencia de medios económicos y de profesionales cualificados para asumir esta compleja tarea.

\section{Enfoque Institucional de la Orientación en Venezuela}

En 1963, la Orientación Educativa se institucionaliza en Venezuela mediante la creación del Servicio Nacional de Orientación (SNOME) por parte del Ministerio. El Servicio dirigió sus esfuerzos hacia la capacitación de los profesionales. Se inicia la formación sistemática de orientadores, se define una metodología de trabajo, buscando unificar las dimensiones organizativa y funcional de los departamentos de orientación y laboratorios psicopedagógicos existentes en el área metropolitana (Cárdenas, 1997).

A partir de 1974, la política educativa del gobierno, denominada "Revolución Educativa", centró la atención en un objetivo prioritario: la democratización de la enseñanza y la modernización del proceso educativo, apoyándose en el informe de Edgar Faure de la UNESCO "Aprender a ser" (1972), que enfatiza en el desarrollo integral del individuo en su ser personal, social y profesional. Estas ideas se materializaron en una nueva estructura educativa que comportaba cuatro niveles:

- Nivel central, que determina lineamientos generales y pretende adaptarlos a los requerimientos específicos de las distintas regiones.

- Nivel regional, cuya función es la de asesorar, supervisar y evaluar la labor que se ejecuta en el nivel de distrito, teniendo un sentido más operativo.

- Nivel distrital, la función esencial reside en ofrecer apoyo y asesoría a las escuelas del sector.

- Nivel núcleos de orientación, integrados por equipos interdisciplinarios de asesoramiento en los planteles.

En este contexto, los objetivos propuestos en los programas de orientación, coordinados por los centros, resultaban bastante ambiciosos para acometer la realidad educativa del país, originando la consiguiente fractura entre los presupuestos teóricos y la práctica. De hecho, 
aunque la División de Orientación del Ministerio de Educación (DOME) elaboró una serie de programas para que fueran aplicados por los Servicios de Orientación, esta propuesta resultó inoperante, dado que su enfoque no respondía a las necesidades socioculturales reales de las regiones y centros educativos.

En la década de los setenta, se crearon diversos servicios: el Centro Nacional de Asesoramiento Vocacional y Profesional (CENAVOP), el Programa de Orientación Educativa Personal-Social y el Programa de Supervisión y Entrenamiento. Curcho (1984) destaca los objetivos del CENAVOP en materia de orientación: facilitar al individuo la adquisición de información sobre el mundo ocupacional y las oportunidades de estudio; asesorarle sobre el conocimiento de sí mismo y promover el desarrollo de las habilidades y de destrezas necesarias para desempeñar eficientemente los roles de estudiante, trabajador y ciudadano.

Pero estos loables proyectos se quedaron en meras intenciones, debido a falta de Servicios que ofrecieran información y ayuda a los estudiantes, en base a las necesidades reales y potenciales de la población escolar. No obstante, algunos orientadores, comprometidos con su tarea, realizaron un notorio esfuerzo tendente a ofrecer a los estudiantes la información requerida, mostrándoles las diversas vías de formación profesional y posterior inserción laboral.

\section{Marco normativo de la Orientación en Venezuela}

La Orientación Educativa en Venezuela tiene su asidero legal en diversas disposiciones: Ley Orgánica de Educación (1980); Normativo de la Educación Básica (1985); Reglamento del Ejercicio de la Profesión Docente (1991); Diseño Curricular de la Escuela Básica (1997); Reglamento General de la Ley Orgánica de Educación (LOE, 1999); y la propia Constitución de la República Bolivariana de Venezuela (1999) (Carrillo, 2001). En todas ellas, se contempla e insiste en el vínculo entre educación y orientación, estableciendo la necesidad de orientación en los centros educativos, extensiva a los alumnos y agentes educativos. Se considera la orientación como un proceso preventivo, dirigido a atender las necesidades, intereses, motivaciones y potencialidades del alumno.

La Ley Orgánica de Educación de 1980 puso el énfasis en "una educación para el desarrollo de la conciencia ciudadana; la conservación, defensa y mejoramiento del ambiente; la calidad de vida y el uso racional de los recursos naturales". Expresa, como fin esencial, "el desarrollo de la personalidad y la formación de valores y actitudes positivas ante la vida y el medio, mediante la integración de esfuerzos que contribuyan al desarrollo integral del individuo". Todos estos aspectos son inherentes al proceso de orientación, considerada como una ayuda e intervención continua y coordinada desde distintas instancias y personas. El Artículo 21 de la citada Ley hace mención explícita a la orientación cuando dice:

"La Educación Básica tiene como finalidad: contribuir a la formación integral del educando mediante el desarrollo de sus destrezas y de su capacidad científica, técnica, humanística y artística; cumplir funciones de exploración y de orientación educativa y vocacional e iniciarles en el aprendizaje de disciplinas y técnicas que les permitan el ejercicio de una función socialmente útil; estimular el deseo de saber y desarrollar la capacidad de ser de cada individuo de acuerdo con sus aptitudes." 
La mencionada Ley establece el modelo organizativo para el funcionamiento de la orientación:

Niveles organizativos de la orientación en Venezuela (1980)

\begin{tabular}{|l|l|l|}
\hline ÁMBITOS & NIVELES DE INTERVENCIÓN & \multicolumn{1}{c|}{ TIPO DE UNIDAD } \\
\hline AULA & - Tutoría en el aula & - Unidad de Acción Directa \\
\hline ESCUELA & - Consejo Superior de la Comunidad & $\begin{array}{l}\text { - Unidad Organizativa Básica del Centro } \\
\text { Escolar }\end{array}$ \\
\hline SECTOR & $\begin{array}{l}\text { - Núcleo integrado por Equipo } \\
\text { de Apoyo del Distrito Escolar }\end{array}$ & $\begin{array}{l}\text { - Unidad Eje en un Distrito o Sector } \\
\text { Escolar }\end{array}$ \\
\hline
\end{tabular}

Al igual que en el Modelo de Orientación propiciado una década después por la LOGSE (1990), en el Sistema Educativo Venezolano el primer nivel corresponde a la tutoría en el aula, ejercida por el docente en su rol de tutor, quien asume las funciones de prevención, desarrollo, asistencia, ayuda, asesoría y guía de los alumnos, padres y representantes, mediante un trabajo colaborativo. Es decir: "La orientación es tarea de todos los agentes que intervienen en la educación y de manera especial le corresponde al docente el rol de tutororientador" (CERPE, 1985).

El artículo 77 de la LOE venezolana (1980) contempla que "el personal docente estará integrado por quienes ejerzan funciones de enseñanza, orientación, planificación, investigación, experimentación, evaluación, dirección, supervisión y administración". En su desarrollo se advierte que la acción orientadora y tutorial no se contempla al margen de las áreas del curriculo, sino plenamente integrada en las mismas. La tutoría conlleva diversas funciones: organización y dinamización del grupo-clase, motivación para el aprendizaje, negociación de roles, resolución de conflictos en el aula, promoción de las relaciones humanas etc., acciones que indudablemente se hallan al servicio del curriculo, que constituye el marco de toda la práctica docente y, por tanto, de la acción tutorial.

En el segundo nivel, representado por el Consejo Superior del Centro, a la orientación le corresponde dar apoyo a la comunidad educativa y asesorar al docente para que pueda asumir la función tutorial con plenas garantías. Para este nivel, se establece que "en todos los grados y actividades de Educación Básica, los órganos de la comunidad educativa atenderán la formación de hábitos y formas de comportamiento de los alumnos, a fin de propiciar un mejor ajuste con su ambiente familiar, social y natural" (artículo 23).

El tercer nivel afecta al Equipo de Apoyo del Distrito Escolar, estando integrado por un equipo multidisciplinar que tiene como principal función prestar apoyo y asesoramiento a los centros sobre la función tutorial, los proyectos curriculares y otros necesidades emanadas de la comunidad educativa.

En 1985, la Orientación, como proceso preventivo y de desarrollo, es retomada en el Normativo de la Escuela Básica, donde contempla, de manera explícita, la asesoría y ayuda al alumno en atención a las necesidades, intereses, potencialidades, limitaciones y a las particularidades de su entorno. Se establecen las pautas de funcionamiento de los Servicios socioeducativos, dentro de los cuales se incluyen los Programas de orientación educativa. En esta época, a raíz de la implantación de la Escuela Básica en todo el país, se promueve el 
desarrollo de planes y estrategias de orientación conjuntamente con los Núcleos Integrales de Bienestar Estudiantil (NIBE), organismos que incluyen los Servicios socioeducativos de salud, orientación y trabajo (Graterol, 1994).

Según Calonge (1988), el Normativo propone diversas líneas ejecutoras de la Orientación Educativa, tales como:

- Uso del tiempo libre. Desarrollo de planes dirigidos a promover en el aula y en la familia el aprovechamiento del tiempo libre, mediante actividades culturales, deportivas y recreativas.

- La Familia. Promover la integración familia-escuela, creando condiciones afectivas para mantener y estrechar los vínculos de seguridad, protección, estímulo, amor y comprensión hacia los niños.

- Desarrollo del autoconcepto. Contribuir al conocimiento de sí mismo, mediante la participación en actividades de orientación individual y grupal.

- Desarrollo vocacional. Integrar la toma de decisiones en el currículo escolar, mediante estrategias tendentes a la promoción y desarrollo de habilidades, actitudes e intereses, con el fin de que el alumno asuma sus propias decisiones vocacionales.

Estas líneas representan una guía eficiente para cualquier Programas de Orientación Educativa. Se insiste en la presencia del orientador especialista en el Consejo Superior de la Comunidad y en la Junta Directiva, con el fin de concienciar, motivar y asesorar sobre actividades escolares y de aprendizaje. Para la organización administrativa y funcional se crean los Núcleos de orientación. Cada Núcleo está integrado por dos o más orientadores, que tiene su sede en una de las Escuelas Básicas del Distrito Escolar, asumiendo la asesoría de todas las Escuelas del Sector.

Con relación a la formación del docente, las Resoluciones Ministeriales $12^{\mathrm{a}}(1985)$ y $1^{\mathrm{a}}$ (1996) plantean que el perfil del profesorado debe responder a dos componentes: el profesional y el de la personalidad. El primero contempla las funciones y tareas que ha de desempeñar el profesor como formador. Específicamente, se refiere al dominio de las competencias en cinco ámbitos: orientador, evaluador, planificador, administrador y promotor de cambios sociales. El perfil de la personalidad se refiere a los rasgos, actitudes y habilidades sociales que debe tener para promover en el alumno el desarrollo integral en todas sus dimensiones (Terán 2002).

Como podemos constatar, la orientación y la formación de orientadores ha estado presente en diversas normativas emanadas del Ministerio de Educación Venezolano, con aspectos que de alguna forma se han anticipado a la LOGSE (1990), como los tres niveles de la orientación o la necesidad intervenir conjuntamente con los demás agentes educativos.

Pero a pesar de las buenas intenciones reflejadas en las mencionadas leyes, la realidad educativa venezolana es bien distinta, debido a que la orientación está en "crisis", a causa del limitado apoyo económico y a la escasa importancia que le atribuye el Estado a los programas de orientación en los centros escolares. Todo ello sumado a la incidencia de factores, tales como el aumento significativo de niños escolarizados, o el solapamiento de la orientación con otras estructuras, al ubicarla dentro de los Servicios Socioeducativos, restándole importancia a la acción tutorial y al Consejo Superior de la comunidad educativa del centro escolar. Estos escollos hacen bastante inoperantes los principios que presiden la normativa sobre orientación en Venezuela. 


\section{Perspectiva actual e integradora de la orientación en el currículo}

El Modelo Curricular (1997), propuesto por el Ministerio de Educación venezolano, constituye un referente teórico que sustenta la Reforma de todo el sector educativo, iniciada en el Nivel Escolar Básico. Mediante su aplicación, se aspira lograr una articulación con los otros niveles del Sistema y con el sector externo (M.E., 1997).

La perspectiva curricular venezolana, al igual la española, ha depositado su confianza en los ejes transversales, como vías para potenciar la formación en dimensiones educativas relevantes. Según Odreman (1997), El Proyecto Educativo Nacional aborda la acción educativa a partir de ejes transversales profundamente éticos, humanísticos y científicos, los cuales favorecen la formación integral y la construcción autónoma de los aprendizajes. En tal sentido, los ejes transversales constituyen temas recurrentes que emergen de la realidad social, entretejidos en cada una de las áreas del currículum, convirtiéndose en los fundamentos para la práctica pedagógica, al integrar los campos del ser, saber, hacer y convivir, a través de conceptos, procesos, valores y actitudes que orientan la enseñanza y el aprendizaje (Molina, 2000) En consecuencia, no pueden considerarse como contenidos paralelos a las áreas, sino como medios que conducen a un aprendizaje que propicia la formación científica-humanística, ético-moral del ser humano, en consonancia con su realidad social.

La filosofía que preside el nuevo Diseño Curricular de la Educación Básica centra su atención en la integridad del sujeto en formación, asumiendo como postulados una escuela comprometida con el alumno y la comunidad, una educación para la vida, centrada en valores, y una práctica pedagógica dirigida a la prevención de conflictos y al autodesarrollo con el fin de que el sujeto alcance su plena autonomía.

Según Peñaloza (1995), la Orientación Educativa se constituye como un eje, cimentado en un sistema de valores, sobre el que se fundamenta la acción educativa. El eje transversal "valores" contempla principios éticos, como la responsabilidad, el trabajo, la solidaridad y la autonomía. La dimensión axiológica implica un proceso de reflexión y diálogo, de acuerdos y compromisos que deben contemplarse en el Proyecto Pedagógico de plantel o centro y deben materializarse en el aula. Es, precisamente este último nivel el que reclama una atención personalizada del alumno.

La reforma venezolana incide en el carácter integrador y vitalista del currículo. Esto conlleva la incorporación de determinados elementos curriculares o ejes transversales, vinculados a las necesidades que la sociedad demanda, como la educación para la paz y para el desarrollo, la educación para la salud, la educación para la igualdad entre los sexos, la educación ambiental, la educación del consumidor y la educación familiar, todos ellos de vital importancia (García et al., 1997).

Los documentos del nuevo diseño reflejan una fuerte influencia europea, especialmente de la Reforma española (LOGSE), en donde los ejes transversales, según Boronat (1997) representan una vía para promover un curriculum integrador, una forma de vincular las disciplinas académicas con la formación humana de los alumnos, tendiendo una especie de puente entre las actividades escolares y la vida misma.

Lo anteriormente expuesto pone de manifiesto la gran responsabilidad que el profesor tutor tiene que asumir en estos tiempos. Debe estar preparado para afrontar con profesiona- 
lidad los nuevos desafíos y cambios que propone la Reforma curricular, que concibe al ser humano desde una perspectiva holística, como un ser integral capaz de lograr el máximo desarrollo de sus potencialidades.

La Reforma española (1990), refrendada en una Ley Orgánica, ha generado numerosos foros de discusión e investigaciones sobre las bondades y debilidades respecto a su filosofía y aplicación, y ha sido objeto de un seguimiento continuo en los medios de comunicación (Turiño, 2003). Pero este debate no solo se ha suscitado en nuestro país, sino que está presente en muchos foros científicos, allende los mares. En tal sentido, la filosofía de la LOGSE preside la política educativa de muchos países hispanoamericanos. Todos ellos consideran que la orientación y la transversalidad son los instrumentos idóneos para conseguir que los cambios preconizados no se queden en lo superficial. En Venezuela, desde el inicio del mandato de Caldera, en 1994, como ministro de educación, se han creado múltiples foros para analizar la filosofía y estructura de su Reforma. Se ha contado con el asesoramiento de expertos españoles para realizar las adaptaciones curriculares pertinentes, la acomodación de los textos escolares, y actualmente se están implementando programas y experiencias desarrolladas con éxito en España.

Dado que la LOGSE ha sido un referente básico para el diseño de la educación venezolana, no queremos finalizar este apartado sin aludir, de forma sucinta, a la situación de la orientación en la normativa actual española. A lo largo del discurso hemos podido constatar la presencia y el reconocimiento de la orientación en la LOGSE (1990), y en la efímera Ley Orgánica de la Calidad de la Educación (LOCE, 2002). En ambas normativas se contempla: "la orientación y la atención psicopedagógica como principio fundamental en el desarrollo de la actividad educativa". En cambio, en el Anteproyecto de la Nueva Ley Orgánica de Educación (LOU, 2005), pendiente de aprobar por el Parlamento, esta dimensión educativa ha sufrido un franco retroceso, puesto que en esta ley marco no se citan, de forma explícita, ni los departamentos de orientación ni determinadas funciones orientadoras (atención psicopedagógica, orientación educativa y profesional, consejo o informe orientador, planes de acción tutorial en centros, etc.), aspectos que favorecen una educación más personalizada, en su doble dimensión individual y sociocultural. Ante este fragante olvido, diversas asociaciones, colectivos y quienes nos sentimos identificados con la orientación, hemos denunciado este hecho, para que se le devuelva a la orientación el status que le corresponde. Sería imperdonable que habiendo sido la normativa española un buen referente para varios países latinoamericanos, la orientación en la nueva ley cayera en el olvido. Abogamos, pues, para que esta laguna sea subsanada antes de su aprobación definitiva, para que de nuevo la presencia y desarrollo de la orientación sea un hecho.

\section{A modo de conclusión}

Los orígenes de la orientación en Venezuela están marcados por una honda influencia española, debido a que conocidos especialistas españoles en el campo de la orientación, a causa de su exilio a diversos países hispanoamericanos, dejaron sentir su influencia en este campo y sus ideas fueron muy bien acogidas en el sistema educativo y laboral venezolano.

Pero como en España, la orientación en Venezuela ha estado a merced de los avatares políticos. De hecho, los avances logrados en los primeros años sufrieron un franco retroce- 
so con la llegada de la dictadura, dando lugar a un periodo de oscurantismo. Fruto de ello fue el retroceso que sufrió el sistema educativo, en general, y la función orientadora, de forma más concreta.

En 1985, la Orientación, como proceso preventivo y de desarrollo, es retomada en el Normativo de la Escuela Básica, donde aparece, de manera explícita, la asesoría y ayuda al alumno en atención a sus necesidades, intereses, potencialidades y limitaciones, así como a las particularidades de su entorno. Esta ley plantea como líneas ejecutoras de la orientación: el uso del tiempo libre, la integración familia-escuela, el desarrollo del autoconcepto y la incorporación de su dimensión profesional en el curriculo. En posteriores disposiciones legales, emanadas de la política educativa venezolana, se reconoce la importancia de la orientación, si bien no se arbitran los medios necesarios para su aplicación.

Recientemente, estando muchos países inmersos en significativos cambios educativos, se están promoviendo amplios debates en torno a sus respectivas Reformas. En el caso venezolano, desde 1994 se han creado múltiples foros de discusión en torno a los fundamentos y estructura de su Sistema Educativo. Fruto de ello ha sido el reconocimiento de la Orientación Educativa como un eje transversal, cimentado en un sistema de valores en torno a la acción educativa. Asimismo, se reconoce la presencia de la orientación en los tres niveles curriculares. En el tercer nivel se aboga por una dimensión personalizada de la orientación, centrada en las necesidades e intereses del alumno.

Lo anteriormente expuesto pone de manifiesto la importancia que se le concede a la orientación. Pero para que las buenas intenciones lleguen a ser operativas, es necesario que el Estado dote a los centros de los medios suficientes para que pueda ponerse la práctica orientadora con total garantía. Pero también se hace imprescindible que la Universidad reconozca la trascendencia de esta función y asuma el compromiso de contemplar en sus planes de estudio la formación y cualificación de los orientadores.

En la actualidad, ante los cambios políticos, sociales y educativos que estamos viviendo en muchos países, se hace necesario que la orientación esté presente con pleno derecho en las políticas educativas, tratando de responder a algunos fenómenos, propios de nuestro tiempo, como la pérdida de valores, el fracaso escolar, la interculturalidad, la desestructuración familiar, ect., problemas que se agudizan en el caso concreto de América Latina, debido a los acusados contrastes, que inevitablemente desembocan en una mayor desigualdad social, cultural y educativa.

Ante estos hechos, se hace necesaria una atenta reflexión sobre el futuro de estos países, tratando de buscar los instrumentos más idóneos para generar los cambios desde la educación, ofreciendo a los escolares el encuentro con una mano extendida que les ayude a construir sus proyectos de vida y a lograr su desarrollo personal y social, razón de ser de la orientación. Cerramos esta comunicación con la siguiente reflexión, planteada en el Congreso Mundial de Orientación (Caracas, 2000), donde se pone de manifiesto la importancia y trascendencia de la tarea orientadora en la sociedad latinoamericana:

"los orientadores no estamos solos emprendiendo las tareas de hacer una educación general
más humana para profesores, estudiantes y toda la sociedad; los orientadores en el ámbito
latinoamericano y mundial hemos comenzado a darnos cuenta de la trascendencia de nuestro
accionar en las perspectivas de la formación del ser humano latinoamericano. En un mundo 
en procesos de acelerados cambios, el ser humano está expuesto a infinidad de situaciones que, aun cuando no logre superar, son parte de su realidad; también lo son sus capacidades y limitaciones personales las que, por falta de oportunidades, a veces no alcanza a conocer y valorar" (p. 2).

\section{Referencias bibliográficas}

Benavent, J. A. (1996). La Orientación Psicopedagógica en España. Desde sus orígenes hasta 1939. Valencia: Promolibro.

Boronat, J. (1997). "Enfoque interdisciplinar en la formación del profesorado sobre los temas transversales". En Asociación Española de Orientación y Psicopedagógica (Comp.) La orientación educativa y la intervención psicopedagógica integrada al curriculo. Valencia: AEOP. pp. 292295.

Calonge, S. (1988). Tendencias de la Orientación en Venezuela. Caracas: Cooperativa Laboratorio Educativo.

Cárdenas, L. (1997). La Educación en Venezuela. Caracas: Ministerio de Educación.

Carrillo, R. (2001). Prospectiva de la Orientación en Venezuela en el Marco de la Reforma Curricular. Carabobo: UC.

Centro Educativo de Reflexión y Planificación. (1985). La Orientación. Caracas: CERPE.

Congreso Mundial de Orientación en Venezuela (2000). Manifiesto de los Orientadores (documento accesible en File://A:Manifiesto de los orientadores. htm).

Curcho, A. (1984). La Orientación Educativa. Caracas: UNA.

Faure, E. et al. (1972). Aprender a ser. Madrid: Alianza Universidad/ UNESCO.

García, J. et al. (1997). La Educación en Valores. Un reto compartido. Madrid: Anaya.

Graterol, S. (1994). Orientación Escolar en la Escuelas Básica. Barinas: Unellez.

Ministerio de Educación. (1980). Ley Orgánica de Educación. Dirección Sectorial y Presupuesto. Caracas: ME.

Ministerio de Educación. (1985). Normativo de la Educación Básica. Dirección Sectorial y Presupuesto. Caracas: ME.

Ministerio de Educación. (1991). Reglamento del Ejercicio de la Profesión Docente. Dirección Sectorial y Presupuesto Caracas: ME.

Ministerio de Educación. (1997). Curriculo Básico Nacional Nivel de Educación Básica. Dirección Sectorial y Presupuesto. Caracas: ME.

Ministerio de Educación y Ciencia (1990). Ley de Ordenación General del Sistema Educativo. Madrid: MEC

Ministerio de Educación y Cultura (2002). Ley Orgánica de Calidad de la Educación. Madrid: MEC.

Ministerio de Educación y Cultura (2005). Anteproyecto de la Ley Orgánica de Educación. Madrid: MEC.

Molina, D. (2000). La Orientación como Práctica Pedagógica. Material de apoyo al subproyecto. Barinas: Unellez.

Odreman, N. (1997). El Proyecto Educativo Nacional. Caracas: ME.

Peñaloza, W. (1995). Currículo Integral. Maracaibo: EDILUZ.

Prieto, A. (2000). Formación y capacitación del docente. Barquisimeto: UPEL. 
Terán, I. (2002). Rol del profesor como orientador en la Escuela Básica. Barinas: Unellez.

Turiño, J.M. (2003). Estudio de la problemática del profesorado en la implantación de la Reforma Educativa en España (1990-2000). Tesis doctoral. Universidad de Valladolid.

Fecha de recepción: 20-04-04

Fecha de revisión: 10-10-05

Fecha de aceptación: 19-10-05 